\title{
Vessel wall MRI characteristics of endovascularly treated aneurysms: association with angiographic vasospasm
}

\author{
*Mahmud Mossa-Basha, MD,', Thien J. Huynh, MD, MSc,, Daniel S. Hippe, MS, ${ }^{1}$ Peter Fata, MD,1 \\ Ryan P. Morton, MD, ${ }^{2}$ and Michael R. Levitt, MD ${ }^{1-3}$ \\ Departments of ${ }^{1}$ Radiology, ${ }^{2}$ Neurosurgery, and ${ }^{3}$ Mechanical Engineering, University of Washington, Seattle, Washington
}

OBJECTIVE The aim of this paper was to evaluate the association between intracranial vessel wall MRI enhancement characteristics and the development of angiographic vasospasm in endovascularly treated aneurysm patients.

METHODS Consecutive cases of both ruptured and unruptured intracranial aneurysms that were treated endovascularly, followed by intracranial vessel wall MRI in the immediate postoperative period, were included. Two raters blinded to clinical data and follow-up imaging independently evaluated for the presence, pattern, and intensity of wall enhancement. Development of angiographic vasospasm was independently evaluated. Delayed cerebral ischemia; cerebral infarct; procedural details; and presence and grade of subarachnoid, parenchymal, and intraventricular hemorrhage were evaluated. Statistical associations were determined on a per-vessel segment and per-patient basis.

RESULTS Twenty-nine patients with 30 treated aneurysms ( 8 unruptured and 22 ruptured) were included in this study. Interobserver agreement was substantial for the presence of enhancement $(\kappa=0.67)$ and nearly perfect for distribution $(\kappa=0.87)$ and intensity $(\kappa=0.84)$ of wall enhancement. Patients with ruptured aneurysms had a significantly greater number of enhancing segments than those with unruptured aneurysms (29.9\% vs $7.2 \%$; OR 5.5, 95\% Cl 2.2-13.7). For ruptured cases, wall enhancement was significantly associated with subsequent angiographic vasospasm while controlling for grade of hemorrhage (adjusted OR 3.9, 95\% CI 1.7-9.4). Vessel segments affected by balloon, stent, or flowdiverter use demonstrated greater enhancement than those not affected (OR 22.7, 95\% Cl 5.3-97.2 for ruptured; and OR $12.9,95 \%$ Cl 3.3-49.8 for unruptured).

CONCLUSIONS Vessel wall enhancement after endovascular treatment of ruptured aneurysms is associated with subsequent angiographic vasospasm.

https://thejns.org/doi/abs/10.3171/2018.4.JNS172829

KEYWORDS vessel wall MRI; vasospasm; aneurysm; vascular disorders

\section{A} NEURYSMAL subarachnoid hemorrhage (aSAH) occurs in 9-14 per 100,000 population in the United States. ${ }^{2}$ Up to two-thirds of patients with aSAH develop angiographic vasospasm and one-third develop delayed cerebral ischemia (DCI), leading to poor outcomes. ${ }^{4,10}$ Although the precise pathophysiology of vasospasm is incompletely understood, previous studies have demonstrated that angiographic vasospasm is induced by adjacent aSAH and correlates with the volume of adjacent hemorrhage. ${ }^{8,17}$ Intracranial vessel wall MRI (IVWM) has emerged as a useful technique to characterize intracranial vasculopathies, particularly inflammatory changes in the vessel wall, ${ }^{12-15}$ and may more directly identify vascular inflammation that may be associated with subsequent vasospasm in the setting of aSAH..$^{11,16}$ The purpose of our study was to characterize IVWM findings in patients with acute endovascularly treated ruptured and unruptured aneurysms, characterize the IVWM features of different treatment techniques, and determine any association with angiographic vasospasm.

\section{Methods}

\section{Patient Cohort}

After institutional review board approval, consecutive patients with a ruptured or unruptured intracranial aneu-

ABBREVIATIONS ACA = anterior cerebral artery; aSAH = aneurysmal SAH; $\mathrm{DCl}=$ delayed cerebral ischemia; GEE = generalized estimating equations; ICA = internal carotid artery; IVWM = intracranial vessel wall MRI; MCA = middle cerebral artery; SAH = subarachnoid hemorrhage; SPACE = sampling perfection with application of optimized contrast enhancement; TCD $=$ transcranial Doppler.

SUBMITTED November 9, 2017. ACCEPTED April 5, 2018.

INCLUDE WHEN CITING Published online September 21, 2018; DOI: 10.3171/2018.4.JNS172829.

${ }^{*}$ M.M.B. and T.J.H. contributed equally to this work. 
rysm treated by a vascular neurosurgeon (M.R.L.) via an endovascular approach were prospectively recruited for this study with waiver of written consent. Verbal consent was obtained in all patients per institutional review board approval. All patients underwent routine clinical MRA with contrast, with IVWM after endovascular aneurysm treatment. Clinical data were recorded by a vascular neurosurgeon in a prospectively maintained institutional IVWM database and included patient demographic data (age, sex, date of birth) and comorbidities (vascular risk factors, stimulant and anticoagulant medication use), antiinflammatory medication use, subarachnoid hemorrhage (SAH) onset, treatment and imaging dates, and baseline CT imaging features, specifically the modified Fisher score $^{8}$ and presence/absence of intraventricular hemorrhage and parenchymal hemorrhage.

\section{MRI Protocol}

Imaging was performed using a 3.0-T Magnetom Trio (Siemens); after the upgrade, a 3.0-T Magnetom Prisma MR system (Siemens) using a 20-channel neurovascular coil was used. As the study spanned the period while our institution transitioned from 2D T1-weighted TSE (turbo spin echo) to 3D T1-weighted SPACE (sampling perfection with application of optimized contrast enhancement) utilization, patients underwent a combined 2D and 3D IVWM protocol, with 3D T1-weighted SPACE before and after contrast administration added later to the protocol. Intravenous gadoteridol (Bracco Pharmaceuticals) was administered on a weight-based protocol at $0.1 \mathrm{mmol} / \mathrm{kg}$, and, after a 5-minute pause, T1-weighted SPACE postcontrast imaging was performed. Imaging protocols are given in Table 1.

\section{IVWM Analysis}

Two board-certified neuroradiologists with limited experience in IVWM interpretation underwent training through review of a packet of selected IVWM articles, as well as group review of 12 IVWM cases (not included in the current study) selected from the institutional database while supervised by a more senior board-certified neuroradiologist with 8 years of experience interpreting IVWM studies. These 2 raters, blinded to patient clinical information and CT and follow-up imaging, independently evaluated the intracranial intradural arterial segments of the circle of Willis using IVWM. Specifically, the bilateral supraclinoid and terminal internal carotid arteries (ICAs), middle cerebral arteries (MCAs; $\mathrm{M}_{1}$ and $\mathrm{M}_{2 / 3}$ segments), anterior cerebral arteries (ACAs; $\mathrm{A}_{1}$ and $\mathrm{A}_{2 / 3}$ segments), posterior cerebral arteries $\left(\mathrm{P}_{1}\right.$ and $\mathrm{P}_{2 / 3}$ segments), vertebral arteries $\left(\mathrm{V}_{4}\right.$ segment), and the basilar artery (maximum of 19 segments per patient) were evaluated. Segments were evaluated for the presence of wall enhancement (greater than normal wall enhancement on postcontrast imaging compared with that on precontrast imaging), intensity of enhancement $(0$, no enhancement; mild, enhancement less than that of the pituitary infundibulum; and moderate, enhancement greater than that of the pituitary infundibulum) ${ }^{14}$ pattern of wall involvement (concentric, $\geq 50 \%$ circumferential involvement; eccentric, $<50 \%$ circum- ferential involvement), ${ }^{6}$ and degree of stenosis (WASID [Warfarin-Aspirin Symptomatic Intracranial Disease] criteria $^{18}$ ). Both 2D and 3D IVWM studies were performed, and correlation with contemporary MRA was allowed. For patients with only 2D T1-weighted IVWM studies, only vessels in the scan field of view with scan planes orthogonal to the lumen were evaluated, although multiple planes of imaging were performed to best assess the circle of Willis branches in their full extent. After independent evaluation, any evaluation disagreements between the 2 readers were resolved by the third expert IVWM neuroradiologist, who was similarly blinded to clinical information and luminal imaging, as well as the reviews from the other raters. Areas of preexisting stenosis on the baseline study present prior to follow-up vasospasm evaluation studies were recorded and categorized as early vasospasm ${ }^{1}$ or intracranial atherosclerosis. The presence of intracranial atherosclerosis was determined after full case review and defined as areas of stable stenosis (on multiple followup imaging studies) with eccentric wall thickening with or without enhancement and outward remodeling on IVWM.

\section{Evaluation of Angiographic Vasospasm, Clinical Outcome, and Treatment Approach}

Data regarding utilization of balloon- or stent-assisted coiling or flow-diverting stents were retrospectively identified from electronic medical records and from review of intraprocedural angiographic images. For each treatment, the precise vessel segment in which a balloon or stent was placed was recorded for subsequent correlation with IVWM findings.

According to institutional management guidelines, all patients with aSAH underwent serial neurological examinations and daily transcranial Doppler (TCD) ultrasonography monitoring for vasospasm screening for 14 days after admission. Follow-up CTA or MRA was performed if there was concern for vasospasm based on clinical examination changes and TCD ultrasonography findings. The presence of angiographic vasospasm of any arterial segment was identified on follow-up imaging (CTA, MRA, or angiography) and recorded after chart and imaging review and defined as new $>50 \%$ stenosis compared with that on baseline imaging. If such imaging studies were unavailable, TCD ultrasonography readings were used to identify moderate vasospasm in the MCA territory using established criteria $(>150 \mathrm{~cm} / \mathrm{sec}$ and Lindegaard ratio $>$ 4.5). The presence of vasospasm at the time of IVWM was recorded separately from the segments that subsequently developed future vasospasm.

Long-term clinical and imaging follow-up was reviewed to determine development of subsequent cerebral infarction or DCI; functional outcome at the final clinical evaluation was graded according to the modified Rankin Scale. DCI was defined as a decrease in 2 points in the Glasgow Coma Scale score or new focal neurological deficit lasting at least 1 hour attributed to vasospasm after other complications, such as rebleeding, hydrocephalus, and systemic disorders, were ruled out. ${ }^{19}$ The presence of cerebral infarction was defined as new infarction on the brain CT or MR scan obtained within 6 weeks after aSAH that was not present on routine imaging between 24 and 
TABLE 1. IVWM protocol

\begin{tabular}{lcccc}
\hline & $\begin{array}{c}\text { 3D T1-Weighted SPACE } \\
\text { Pre- \& Postcontrast }\end{array}$ & $\begin{array}{c}\text { 2D T1-Weighted IVWM } \\
\text { Pre- \& Postcontrast }\end{array}$ & $\begin{array}{c}\text { 3D T2-Weighted } \\
\text { SPACE }\end{array}$ & $\begin{array}{c}\text { 3D TOF } \\
\text { MRA }\end{array}$ \\
\hline TR/TE $(\mathrm{msec})$ & $900 / 15$ & $1000 / 10$ & $1000 / 133$ & $22 / 3.69$ \\
\hline In-plane resolution $(\mathrm{mm})$ & $0.56 \times 0.56$ & $0.4 \times 0.35$ & $0.5 \times 0.5$ & $0.3 \times 0.3$ \\
\hline Slice thickness $(\mathrm{mm})$ & 0.56 & 2 & 0.5 & 0.5 \\
\hline Flip angle $\left({ }^{\circ}\right)$ & Alternating & 90 & Alternating & 18 \\
\hline Field of view $\left(\mathrm{mm}^{3}\right)$ & $180 \times 180$ & $180 \times 158$ & $200 \times 200$ & $205 \times 184$ \\
\hline Matrix & $320 \times 320$ & $450 \times 450$ & $384 \times 384$ & $384 \times 384$ \\
\hline GRAPPA & 2 & 1 & & 2 \\
\hline Averages & 1 & 4 & 2 & 1 \\
\hline Scan time & $8: 08$ & 36 sec/slice & $7: 28$ & $5: 40$ \\
\hline
\end{tabular}

GRAPPA = generalized autocalibrating partial parallel acquisition; TOF = time of flight.

* Scan time is presented as minutes:seconds unless indicated otherwise.

48 hours after early aneurysm treatment, and not attributable to other causes. ${ }^{19}$ Use of endovascular intervention for vasospasm treatment was also identified.

\section{Statistical Analysis}

For descriptive statistics, categorical variables are presented as percentages, and continuous variables are presented as medians with IQR. For patient-level analyses, the Wilcoxon signed-rank test and Fisher's exact test were used to compare continuous and categorical variables between groups. For segment-level analyses, logistic regression with generalized estimating equations (GEE) was used to compare binary variables-e.g., presence of IVWM enhancement, concentric involvement, enhancement intensity, and angiographic vasospasm-between groups while accounting for the nonindependence of different vessel segments from the same patient. To eliminate potential confounding effects of stent or balloon use and IVWM enhancement, segments treated with balloon- or stent-assisted coiling or flow-diverting stents were either analyzed separately or excluded from most analyses, unless they were being directly compared with segments not treated with stents or balloons. Interobserver agreement for the presence of wall enhancement, concentric involvement, and enhancement intensity was assessed using Cohen's $\kappa$ statistic. Values of $\kappa$ of $0.0-0.2,0.21-0.4,0.41-0.6$, $0.61-0.8$, and $0.81-1$ were considered poor, fair, moderate, substantial, and excellent agreement, respectively. All statistical analysis was performed in $\mathrm{R}$ version 3.1.1 (R Foundation for Statistical Computing). Statistical significance was defined as $\mathrm{p}<0.05$ for all tests.

\section{Results}

\section{Patient and Aneurysm Characteristics}

Between September 2015 and November 2016, 30 patients with 32 treated aneurysms (23 ruptured and 9 unruptured) were recruited for the study. One patient with a ruptured aneurysm was excluded due to only 3 segments being evaluable (basilar artery and paired $\mathrm{V}_{4}$ ). One unruptured aneurysm was excluded as it was treated 3 months after treating a ruptured aneurysm that was already included in the study. Lastly, 1 patient had 2 unruptured aneurysms treated simultaneously and underwent a single IVWM study and was considered as a single case in the analysis. Thus, 29 patients were included in the data analysis, 22 with ruptured aneurysms and 7 with unruptured aneurysms.

Patient and study characteristics are described in Table 2. The median (IQR) of the maximum dimension of the aneurysms was $6.6 \mathrm{~mm}(5.3-9.3 \mathrm{~mm})$ with no significant difference between ruptured and unruptured aneurysms (median 6.2 vs $7.5 \mathrm{~mm}, \mathrm{p}=0.14$ ). Twelve aneurysms $(40.0 \%)$ were in the anterior communicating artery, 11 $(36.7 \%)$ were in the posterior communicating artery, 5 $(16.7 \%)$ were in the vertebrobasilar system, and $2(6.7 \%)$ were in other segments. A similar proportion of IVWM studies of ruptured and unruptured aneurysms had 3D T1weighted IVWM $(54.5 \%$ vs $57.1 \%, p>0.99)$. IVWM was performed a median (IQR) of 1 day (1-2 days) after treatment $(\mathrm{p}=0.65$ comparing ruptured and unruptured aneurysms). Of the 22 patients with aSAH, $15(68.2 \%)$ had a modified Fisher grade 4 SAH. Three $(13.6 \%)$ patients had grade $1 \mathrm{SAH}$, and $2(9.1 \%)$ patients each had grade 2 or 3 SAH. IVWM was performed a median (IQR) of 3 days (2-5 days) after aSAH. At the time of IVWM, no patients were undergoing therapy for angiographic vasospasm.

\section{IVWM Characteristics of Ruptured and Unruptured Aneurysms}

In total, 514 vessel segments were analyzed, with 15 to 19 segments evaluated per patient (IQR 17-19). Of these, 48 segments had been treated with assisted coiling $(\mathrm{n}=$ 34 balloon and 6 stent) or a flow-diverting stent $(\mathrm{n}=8)$ and 466 were not. Table 3 summarizes IVWM characteristics in segments grouped by aneurysm rupture status and treatment. Among the segments in patients with ruptured aneurysms, those treated with assisted coiling or flow-diverting stents were significantly more likely to demonstrate IVWM enhancement than other segments (90.6\% vs 29.9\%; OR 22.7, 95\% CI 5.3-97.2, p < 0.001). Results were similar in the patients with unruptured aneurysms (50.0\% vs 7.2\%; OR 12.9, 95\% CI 3.3-49.8, p < 0.001). Figure 1 demonstrates arterial wall enhancement associated with prior balloon-assisted coiling. There were 
TABLE 2. Patient, aneurysm, and treatment characteristics

\begin{tabular}{|c|c|c|c|c|}
\hline \multirow[b]{2}{*}{ Variable } & \multirow[b]{2}{*}{ All Studies $(n=29)$} & \multicolumn{2}{|c|}{ Aneurysm Status } & \multirow[b]{2}{*}{$p$ Value* } \\
\hline & & Ruptured $(n=22)$ & Unruptured $(n=7)$ & \\
\hline Age, yrs & $57(49-59)$ & $56(45-59)$ & $58(56-64)$ & 0.36 \\
\hline Female sex & $23(79.3)$ & $17(77.3)$ & $6(85.7)$ & $>0.99$ \\
\hline Diabetes† & $4(14.8)$ & $4(19.0)$ & $0(0.0)$ & 0.55 \\
\hline Hypertension† & $14(56.0)$ & $12(57.1)$ & $2(50.0)$ & $>0.99$ \\
\hline Hyperlipidemia† & $7(26.9)$ & $6(27.3)$ & $1(25.0)$ & $>0.99$ \\
\hline Smoking history $\dagger$ & $16(57.1)$ & $14(66.7)$ & $2(28.6)$ & 0.1 \\
\hline \multicolumn{5}{|l|}{ Medications } \\
\hline Aspirin & $12(41.4)$ & $6(27.3)$ & $6(85.7)$ & 0.011 \\
\hline Other antiinflammatory & $3(10.3)$ & $3(13.6)$ & $0(0.0)$ & 0.56 \\
\hline \multicolumn{5}{|l|}{ Treatment } \\
\hline Coils alone & $8(27.6)$ & $7(31.8)$ & $1(14.3)$ & 0.63 \\
\hline Balloon-assisted coiling & $16(55.2)$ & $13(59.1)$ & $3(42.9)$ & 0.67 \\
\hline Stent-assisted coiling & $3(10.3)$ & $0(0.0)$ & $3(42.9)$ & 0.01 \\
\hline Flow-diverting stent & $4(13.8)$ & $2(9.1)$ & $2(28.6)$ & 0.24 \\
\hline IVWM protocol & & & & $>0.99$ \\
\hline 2D only & $13(44.8)$ & $10(45.5)$ & $3(42.9)$ & \\
\hline $2 D \& 3 D$ & $16(55.2)$ & $12(54.5)$ & $4(57.1)$ & \\
\hline
\end{tabular}

no significant differences in pattern of wall involvement ( $\mathrm{p}$ $=0.83)$ and intensity of enhancement $(\mathrm{p}=0.55)$ between segments treated or not treated with assisted coiling or flow-diverting stent.

Within the 466 segments not treated with assisted coiling or flow-diverting stents, IVWM enhancement was significantly more likely in ruptured aneurysms than unruptured aneurysms (29.9\% vs 7.2\%; OR 5.5, 95\% CI 2.2-13.7, $\mathrm{p}<0.001$ ) (Table 3). There were no significant differences in concentric involvement $(\mathrm{p}=0.19)$ or intensity of enhancement $(p=0.71)$ between these 2 groups. The findings were similar within the 48 segments treated with assisted coiling or flow-diverting stents (Table 3). When analyzed by patient, there was a greater median (IQR) number of enhancing vessel segments per patient for patients with ruptured aSAH than for patients with unruptured aneurysms $(4[1-7]$ vs 1 [0-2], $p=0.035)$.

Among segments in patients with ruptured aneurysms not treated with assisted coiling or flow-diverting stents, the likelihood of IVWM enhancement decreased with increasing time between aSAH and imaging (OR 0.8 per 1 -day increase, $95 \%$ CI $0.7-1.0, \mathrm{p}=0.029$ ). There was a similar association between enhancement and time between aneurysm treatment and imaging among treated

TABLE 3. Enhancement characteristics of vessel wall segments

\begin{tabular}{|c|c|c|c|c|c|c|}
\hline \multirow[b]{3}{*}{ Variable } & \multicolumn{3}{|c|}{$\begin{array}{l}\text { Segments Not Treated w/ Assisted Coiling or } \\
\text { Flow-Diverting Stent }(n=466)\end{array}$} & \multicolumn{3}{|c|}{$\begin{array}{l}\text { Segments Treated w/ Assisted Coiling or } \\
\text { Flow-Diverting Stent }(n=48)\end{array}$} \\
\hline & \multicolumn{2}{|c|}{ Aneurysm Status } & \multirow{2}{*}{$\begin{array}{c}p \\
\text { Value }^{*}\end{array}$} & \multicolumn{2}{|c|}{ Aneurysm Status } & \multirow{2}{*}{$\begin{array}{c}\mathrm{p} \\
\text { Valuex }\end{array}$} \\
\hline & Ruptured $(n=355)$ & Unruptured $(n=111)$ & & Ruptured $(n=32)$ & Unruptured $(n=16)$ & \\
\hline Presence of enhancement & $106(29.9)$ & $8(7.2)$ & $<0.001$ & $29(90.6)$ & $8(50.0)$ & 0.023 \\
\hline Distribution of enhancement $†$ & & & 0.19 & & & 0.21 \\
\hline Concentric & $97(91.5)$ & $6(75.0)$ & & $27(93.1)$ & $6(75.0)$ & \\
\hline Eccentric & $9(8.5)$ & $2(25.0)$ & & $2(6.9)$ & $2(25.0)$ & \\
\hline Intensity of enhancement† & & & 0.71 & & & 0.60 \\
\hline Mild & $69(65.1)$ & $6(75.0)$ & & $21(72.4)$ & $5(62.5)$ & \\
\hline$>$ Mild & $37(34.9)$ & $2(25.0)$ & & $8(27.6)$ & $3(37.5)$ & \\
\hline
\end{tabular}

Values are number of segments (\%) unless otherwise specified.

* Wald test from GEE-based logistic regression comparing segments between patients with ruptured and unruptured aneurysms.

$\dagger$ Among segments with enhancement present. 

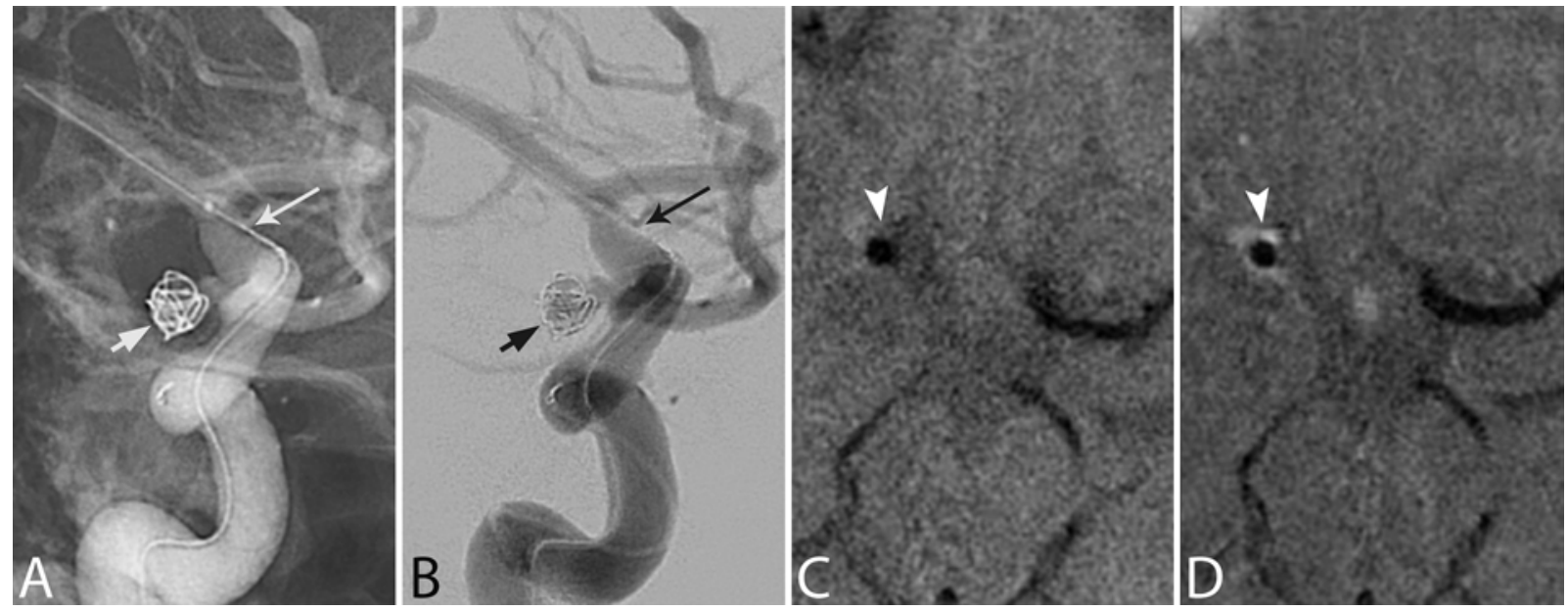

FIG. 1. IVWM enhancement associated with balloon-assisted coiling. Magnified right ICA injection digital unsubtracted (A) and subtracted (B) anteroposterior (AP) angiograms demonstrating coiling of a right posterior communicating artery aneurysm (short arrows) with balloon assistance (long arrows) in the right terminal ICA. Pre- (C) and postcontrast (D) axial IVWM scans obtained on day 1 postcoiling, demonstrating circumferential enhancement involving the right terminal ICA (arrowheads). Note the intrinsic $\mathrm{T} 1$ hyperintense signal adjacent to the artery on $\mathrm{C}$, representing $\mathrm{SAH}$, and note the lack of enhancement in the normal left $\mathrm{M}_{1}$ segment for reference.

segments $(n=48)$, although the association was not statistically significant $(\mathrm{OR} 0.8,95 \%$ CI $0.6-1.1, \mathrm{p}=0.14)$ The likelihood of enhancement was not significantly associated with the use of aspirin $(\mathrm{p}=0.54)$ or any antiinflammatory medication $(\mathrm{p}=0.41)$. The above associations between enhancement, aneurysm rupture status, and treatment were similar with and without adjustment for time to IVWM and medications.

The likelihood of IVWM enhancement varied between the different vessel wall segments among the 355 segments in patients with ruptured aneurysms that were not treated with assisted coiling or a flow-diverting stent $(\mathrm{p}<0.001)$, with the per-vessel prevalence ranging from $34.2 \%$ to $47.4 \%$ in the ICA, ACA, and $\mathrm{M}_{1}$ and from $15.9 \%$ to $22.2 \%$ in $\mathrm{M}_{2 / 3}$ and posterior segments (Table 4). There was no significant difference in terms of the presence of enhancement between the segments imaged with the $2 \mathrm{D}$ T1-weighted IVWM protocol and those imaged with the 3D T1-weighted protocol $(22.4 \%$ vs $35.1 \%, \mathrm{p}=0.24)$.

\section{Association of Angiographic Vasospasm and IVWM Characteristics in aSAH}

Of the 22 aSAH patients, 11 (50.0\%) developed angiographic vasospasm during their admission, including 2 patients who underwent IVWM at 8 and 13 days postaSAH and had angiographic vasospasm at the time of imaging. Of the 15 patients with a modified Fisher grade of 4, $10(66.7 \%)$ developed vasospasm compared with 1 of 7 $(14.3 \%)$ patients with a grade $<4 \mathrm{SAH}(\mathrm{p}=0.063)$. There were 355 vessel segments not treated with assisted coiling or a flow-diverting stent, of which 57 (16.1\%) segments developed vasospasm and 106 (29.9\%) demonstrated IVWM enhancement. Segments with modified Fisher grade 4 SAH were more likely to show enhancement than those with grade < 4 SAH (89/239 [37.2\%] vs 17/116 [14.7\%], p $=0.023)$.
The likelihood of vasospasm was significantly higher in segments with IVWM enhancement than in segments without enhancement (35/106 [33.0\%] vs 22/249 [8.8\%]; OR 5.1, 95\% CI 2.2-11.8, $\mathrm{p}<0.001)$. This association between IVWM enhancement and vasospasm remained statistically significant after adjustment for modified Fisher grade (adjusted OR 3.9, 95\% CI 1.7-9.4, p = 0.005), time from aSAH to IVWM (adjusted OR 5.0, 95\% CI 2.2-11.4, $\mathrm{p}<0.001$ ), or use of antiinflammatory medications (adjusted OR 5.1, 95\% CI 2.0-13.2, $\mathrm{p}=0.001$ ). Similarly, the association between IVWM enhancement and vasospasm remained significant after excluding the 2 patients who demonstrated vasospasm at the time of IVWM (OR 5.4, 95\% CI 1.9-15.9, $\mathrm{p}=0.002)$. When analyzed per patient,

TABLE 4. Frequency of enhancement for each vessel wall segment

\begin{tabular}{lcl}
\hline \multirow{2}{*}{ Segment } & \multicolumn{2}{c}{ By Aneurysm Status* } \\
\cline { 2 - 3 } Supraclinoid ICA & Ruptured & Unruptured \\
\hline ICA terminus & $14 / 37(37.8)$ & $0 / 9(0.0)$ \\
\hline $\mathrm{A}_{1}$ & $14 / 35(40.0)$ & $0 / 9(0.0)$ \\
\hline $\mathrm{A}_{2 / 3}$ & $18 / 38(47.4)$ & $3 / 12(25.0)$ \\
\hline $\mathrm{M}_{1}$ & $13 / 38(34.2)$ & $0 / 12(0.0)$ \\
\hline $\mathrm{M}_{2 / 3}$ & $16 / 40(40.0)$ & $2 / 12(16.7)$ \\
\hline $\mathrm{P}_{1}$ & $7 / 44(15.9)$ & $0 / 14(0.0)$ \\
\hline $\mathrm{P}_{2 / 3}$ & $7 / 40(17.5)$ & $2 / 14(14.3)$ \\
\hline Basilar artery & $9 / 44(20.5)$ & $0 / 14(0.0)$ \\
\hline $\mathrm{V}_{4}$ & $4 / 21(19.0)$ & $1 / 7(14.3)$ \\
\hline
\end{tabular}

Segments treated with assisted coiling or flow-diverting stent were excluded.

* Number of enhancing vessel segments (\%) of the total number of segments. 

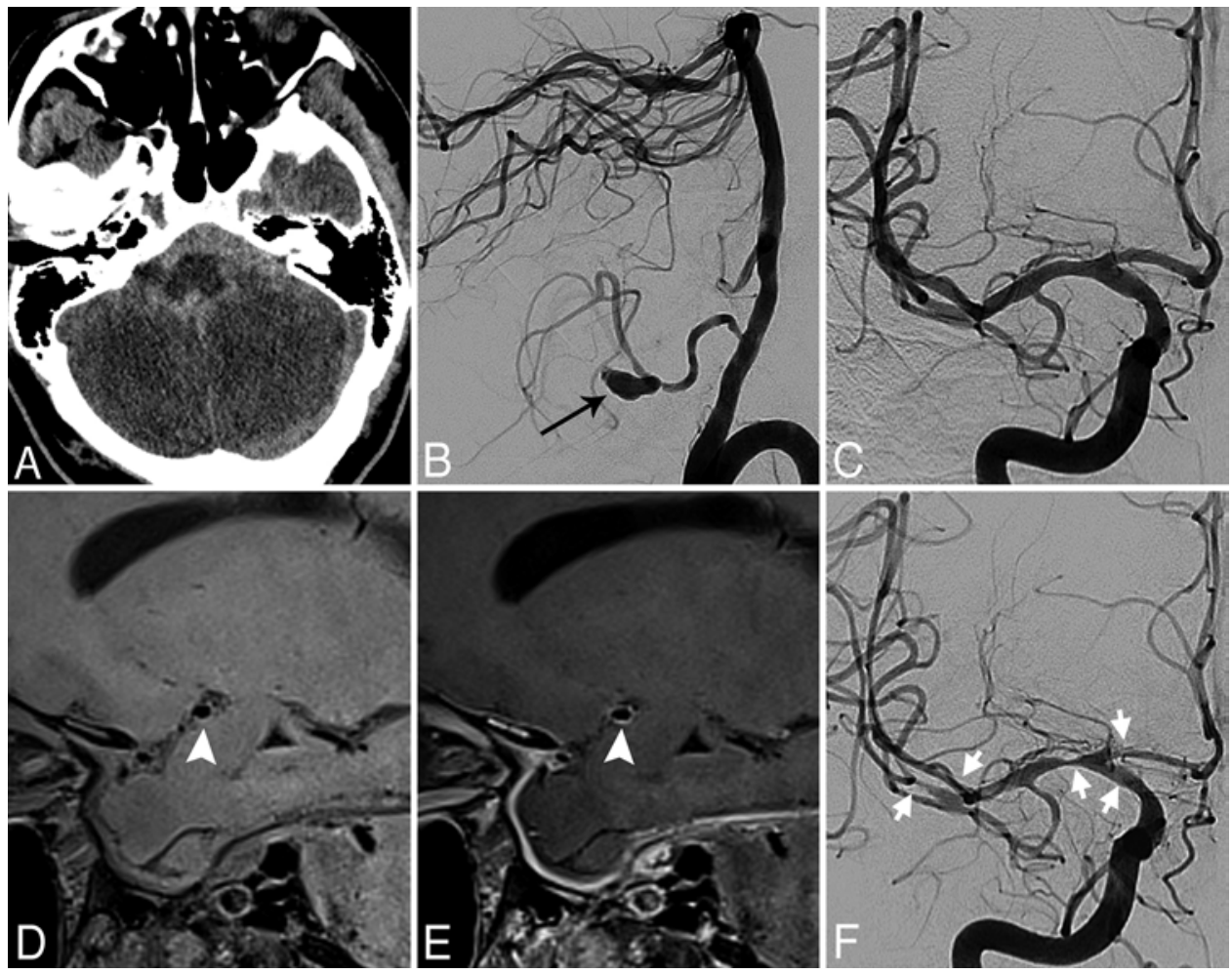

FIG. 2. IVWM enhancement associated with subsequent vasospasm. Modified Fisher grade 4 aSAH seen on a noncontrast head CT scan (A). Digital subtraction angiogram, lateral view, of the left vertebral artery injection (B) showing a prominent ruptured posterior inferior cerebellar artery aneurysm (black arrow). Digital subtraction angiogram, AP view, of the right ICA catheter injection (C) showing normal appearance of the right MCA. Sagittal T1-weighted pre- (D) and postcontrast (E) IVWM scans showing circumferential wall thickening and enhancement of the right MCA (arrowheads). Digital subtraction angiogram, AP view, of the right ICA catheter injection (F) obtained 1 week later, showing severe vasospasm (short arrows) involving multiple arterial segments, including multifocal involvement of the right MCA. Prior to angiography, the patient had developed $\mathrm{DCl}$, and follow-up MRI showed acute infarcts in both MCA territories and the left cerebellum on diffusion-weighted imaging (not shown).

the number of enhancing segments was numerically higher in those who developed angiographic vasospasm compared with those who did not, but the difference was not statistically significant (median [IQR] $5[2-8]$ vs $2[0-6]$, $\mathrm{p}=0.16)$.

The overall sensitivity, specificity, positive predictive value, and negative predictive value on a per-vessel basis of IVWM enhancement for association with angiographic vasospasm were $61.4 \%(35 / 57,95 \%$ CI $44.3 \%-76.1 \%), 76.2 \%$ (227/298, 95\% CI 64.2\%-85.1\%), 33.0\% (35/106, 95\% CI $14.6 \%-58.6 \%)$, and $91.2 \%$ (227/249, 95\% CI 82.1\%-95.9\%), respectively. Of the 106 enhancing vessels, 0 of 9 with an eccentric enhancement pattern developed vasospasm compared with 35 of 97 (36.1\%) with concentric involvement (no statistical test was performed due to the small number of vessels with the eccentric pattern). Enhancement intensity was not significantly different between segments that did and did not develop vasospasm (13/37 [35.1\%] vs 22/69 [31.9\%], $\mathrm{p}=0.80)$. A representative image of IVWM arterial segment enhancement in a patient who subsequently developed vasospasm is demonstrated in Fig. 2.

The 2 aSAH patients with angiographic vasospasm at the time of IVWM had a total of 29 segments reviewed, none of which had prior treatment with assisted coiling/ flow-diverting stent. Of these segments, 10 (34.5\%) dem- onstrated angiographic vasospasm at the time of IVWM. Vasospasm was detected in 8 of $10(80.0 \%)$ segments with IVWM enhancement compared with 2 of 19 (10.5\%) segments without enhancement (no statistical test was performed due to the small number of patients). An example of enhancement at the time of vasospasm is demonstrated in Fig. 3.

\section{DCl and Relationship to IVWM Characteristics in aSAH}

Of 22 patients with aSAH, 6 (27.3\%) developed DCI. Of these, 3 underwent endovascular intervention for vasospasm treatment and 2 had DCI-related infarcts at followup. All 6 of the DCI cases were in patients who demonstrated angiographic vasospasm. The number of enhancing segments was not significantly different between those who did $(\mathrm{n}=6)$ and did not $(\mathrm{n}=16)$ develop DCI (median [IQR] 8 [4-10] vs 4 [2-8], p = 0.23). The number of enhancing segments in those who developed angiographic vasospasm but not DCI $(n=5)$ appeared to be similar to that in those who did develop DCI (median 8 [5-8] vs 8 [4-10], $\mathrm{p}=0.85)$.

\section{Interobserver Agreement}

Interobserver agreement for the presence and absence 

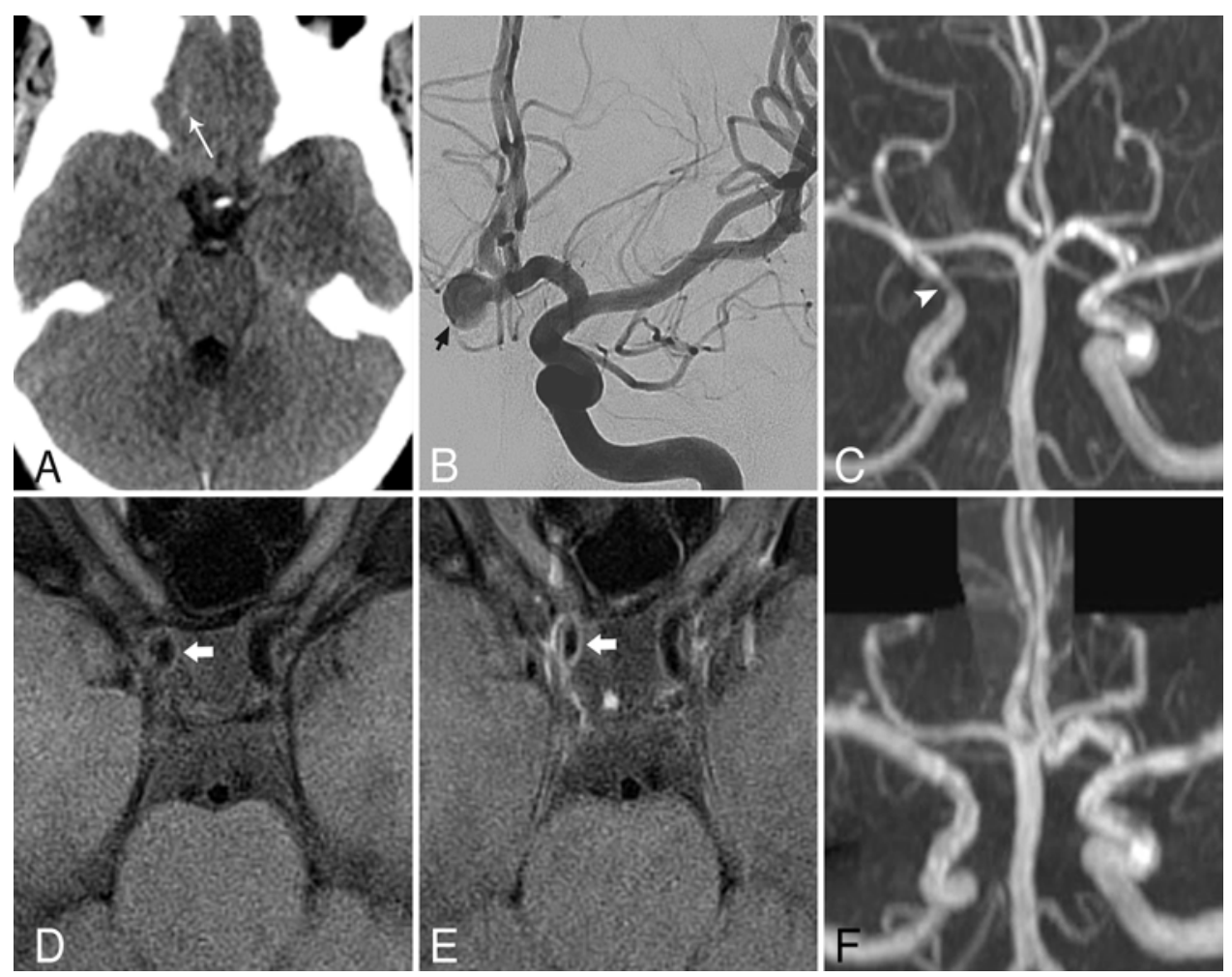

FIG. 3. IVWM enhancement at the time of vasospasm. Axial noncontrast head CT scan (A) showing sulcal SAH (arrow) at the right anterior skull base, corresponding to modified Fisher grade 1 hemorrhage. Digital subtraction angiogram, AP view, the left ICA catheter injection (B), showing a prominent anterior communicating artery aneurysm projecting to the right (short arrow), which was the source of subarachnoid blood. Coronal maximum intensity projection MR angiogram (C) obtained 10 days after treatment, showing focal high-grade stenosis of the right supraclinoid ICA (arrowhead) that was not present on the initial catheter angiogram (not shown). Axial T1-weighted IVWM scans obtained before (D) and after (E) contrast images acquired at the same time as panel $\mathrm{C}$, revealing wall thickening and circumferential enhancement (thick arrows) corresponding to the focus of stenosis. Contemporary diffusion-weighted imaging (not shown) showed a right MCA territory acute infarct. Follow-up MR angiogram (F) acquired 6 months after treatment, showing resolution of stenosis.

of vessel wall enhancement was substantial $(\kappa=0.67,95 \%$ CI 0.52-0.79). Interobserver agreement for distribution $(\kappa$ $=0.87,95 \%$ CI $0.49-1.00)$ and intensity of wall enhancement $(\kappa=0.84,95 \%$ CI $0.44-1.00)$ was excellent among the segments where both observers agreed on the presence of enhancement.

\section{Discussion}

We report the first study to systematically assess IVWM findings in patients after aneurysm treatment, specifically the association of IVWM characteristics with subsequent development of angiographic vasospasm and DCI, as well as procedure-related characteristics. Compared with unruptured aneurysms, there is a significant association between arterial segment wall enhancement and both previous aneurysm rupture and the presence of aSAH. In addition, we found a significant association between arterial segment wall enhancement and subsequent development of substantial angiographic vasospasm within the same segment, as well as a significantly higher number of enhancing segments in patients who developed angiographic vasospasm, which persisted while controlling for the modified Fisher grade of SAH. This study provides the first evidence that IVWM characteristics such as wall enhancement are associated with the development of angiographic vasospasm and may allow for early initiation of preventative therapies to attempt to reduce potential severe morbidity and mortality.

While there was a significant difference in the number of enhancing segments between patients with treated ruptured aneurysms and those with treated unruptured aneurysms, $7.2 \%(8 / 111)$ of untreated arterial segments showed wall enhancement, which was somewhat unexpected. Seven of 8 segments were from 3 patients who were 53-69 years old and had at least one additional vascular risk factor beyond age for atherosclerosis ( 2 patients had 2 additional risk factors). The eighth segment was in a 58 -year-old patient without any additional known vascular risk factors. In addition, each patient had a fixed stenosis involving a separate segment, presumed to represent intracranial atherosclerosis. A possible explanation is that most, if not all, of these lesions may represent nonstenotic atherosclerotic plaques that were not detected on luminal imaging, lesions that have been previously described in the literature. ${ }^{5,20}$

There has been little investigation of IVWM characteristics in the setting of aSAH or vasospasm. A previous 
preliminary study describing IVWM in patients with angiography-negative SAH found that 2 of 11 cases showed multifocal arterial wall enhancement presumed to be secondary to inflammatory changes related to adjacent subarachnoid blood. ${ }^{3}$ We hypothesize that enhancement of the arterial wall in the setting of aSAH likely is related to a combination of underlying inflammation and associated ultrastructural vessel wall changes, including tight junction disruption and endothelial and smooth muscle cell injury? This inflammation is likely the reason for a higher incidence of arterial segment enhancement in patients with ruptured compared with unruptured aneurysms. In addition, segments with inflammatory changes and associated wall enhancement in the setting of aSAH are more likely to progress to vasospasm.

Current predictors of angiographic vasospasm based on initial assessment include the extent of SAH, assessed by grading scales such as the modified Fisher score, as well as clinical factors such as hypertension, cigarette smoking, and left ventricular hypertrophy as detected on electrocardiography. ${ }^{9}$ The most reliably used measure is the extent of SAH; however, evaluation of severity of vasospasm and association with DCI is limited with current markers. For instance, in the current study, one patient who developed ischemic symptoms in the MCA territory and infarct secondary to vasospasm presented with modified Fisher grade $1 \mathrm{SAH}$, which is associated with a low risk of DCI. However, IVWM demonstrated arterial wall enhancement involving the eventually vasospastic segments, indicating that IVWM enhancement measures may be helpful in some circumstances. IVWM can provide a direct measure of arterial inflammatory changes that may better indicate arterial segments at risk for developing angiographic vasospasm. IVWM wall enhancement could also serve as a useful biomarker to evaluate the response to potential new antiinflammatory vasospasm therapies.

To avoid confusing procedure-related wall changes with pathologic changes relating to inflammatory vasculopathy, we separately evaluated for the presence of arterial vessel wall enhancement in vascular segments where endovascular techniques such as balloon- or stent-assisted coiling or flow-diverting stent placement were deployed. We found significant associations between wall enhancement and the affected arterial segments, findings similar to those in a prior study in which stent retriever devices were used for mechanical thrombectomy. ${ }^{16}$ Considering this association, careful assessment of the procedure performed should be taken into account when reviewing IVWM studies, and arterial segments in which these techniques were used should not be considered for the subsequent development of vasospasm.

There are several limitations of the current study. First, the time interval between treatment and imaging was heterogeneous, especially in patients with ruptured aneurysms due to patient stability immediately posttreatment. However, the majority of IVWM studies took place before the typical onset of vasospasm; only 4 of 30 scans were obtained 5 or more days after ictus, and adjusting for the time interval had little effect on the results. Second, the current study addresses angiographic vasospasm and not symptomatic vasospasm or DCI primarily, as the patient population was small and the number of DCI cases was limited for statistical analysis. With larger studies, better evaluation of the correlation between wall enhancement and the development of symptomatic vasospasm or infarct will be possible. Finally, the imaging protocol was not completely consistent throughout the study due to system upgrades. However, this resulted only in the addition of 3D T1-weighted IVWM sequences.

\section{Conclusions}

IVWM wall enhancement preliminarily shows a significant association with subsequent development of angiographic vasospasm on a per-arterial segment basis. With further study, IVWM wall enhancement may prove to be an additional marker for vasospasm that could also track treatment response and provide a new therapeutic target.

\section{Acknowledgments}

Dr. Mossa-Basha and Mr. Hippe received research support from the NIH grant nos. R56 NS092207 01 and R01 NS092207 01A1.

\section{References}

1. Baldwin ME, Macdonald RL, Huo D, Novakovic RL, Goldenberg FD, Frank JI, et al: Early vasospasm on admission angiography in patients with aneurysmal subarachnoid hemorrhage is a predictor for in-hospital complications and poor outcome. Stroke 35:2506-2511, 2004 (Erratum in Stroke 36:175, 2005)

2. Connolly ES Jr, Rabinstein AA, Carhuapoma JR, Derdeyn CP, Dion J, Higashida RT, et al: Guidelines for the management of aneurysmal subarachnoid hemorrhage: a guideline for healthcare professionals from the American Heart Association/American Stroke Association. Stroke 43:1711-1737, 2012

3. Coutinho JM, Sacho RH, Schaafsma JD, Agid R, Krings T, Radovanovic I, et al: High-resolution vessel wall magnetic resonance imaging in angiogram-negative non-perimesencephalic subarachnoid hemorrhage. Clin Neuroradiol 27:175183, 2017

4. Crowley RW, Medel R, Dumont AS, Ilodigwe D, Kassell NF, Mayer SA, et al: Angiographic vasospasm is strongly correlated with cerebral infarction after subarachnoid hemorrhage. Stroke 42:919-923, 2011

5. de Havenon A, Yuan C, Tirschwell D, Hatsukami T, Anzai Y, Becker K, et al: Nonstenotic culprit plaque: the utility of high-resolution vessel wall MRI of intracranial vessels after ischemic stroke. Case Rep Radiol 2015:356582, 2015

6. Dieleman N, Yang W, Abrigo JM, Chu WC, van der Kolk AG, Siero JC, et al: Magnetic resonance imaging of plaque morphology, burden, and distribution in patients with symptomatic middle cerebral artery stenosis. Stroke 47:17971802,2016

7. Findlay JM, Weir BK, Kanamaru K, Espinosa F: Arterial wall changes in cerebral vasospasm. Neurosurgery 25:736746, 1989

8. Frontera JA, Claassen J, Schmidt JM, Wartenberg KE, Temes $\mathrm{R}$, Connolly ES Jr, et al: Prediction of symptomatic vasospasm after subarachnoid hemorrhage: the modified fisher scale. Neurosurgery 59:21-27, 2006

9. Inagawa T: Risk factors for cerebral vasospasm following aneurysmal subarachnoid hemorrhage: a review of the literature. World Neurosurg 85:56-76, 2016

10. Macdonald RL: Delayed neurological deterioration after subarachnoid haemorrhage. Nat Rev Neurol 10:44-58, 2014 
11. Mandell DM, Mossa-Basha M, Qiao Y, Hess CP, Hui F, Matouk C, et al: Intracranial vessel wall MRI: principles and expert consensus recommendations of the American Society of Neuroradiology. AJNR Am J Neuroradiol 38:218-229, 2017

12. Mossa-Basha M, Alexander M, Gaddikeri S, Yuan C, Gandhi D: Vessel wall imaging for intracranial vascular disease evaluation. J Neurointerv Surg 8:1154-1159, 2016

13. Mossa-Basha M, de Havenon A, Becker KJ, Hallam DK, Levitt MR, Cohen WA, et al: Added value of vessel wall magnetic resonance imaging in the differentiation of moyamoya vasculopathies in a non-Asian cohort. Stroke 47:17821788,2016

14. Mossa-Basha M, Hwang WD, De Havenon A, Hippe D, Balu $\mathrm{N}$, Becker KJ, et al: Multicontrast high-resolution vessel wall magnetic resonance imaging and its value in differentiating intracranial vasculopathic processes. Stroke 46:1567-1573, 2015

15. Mossa-Basha M, Shibata DK, Hallam DK, de Havenon A, Hippe DS, Becker KJ, et al: Added value of vessel wall magnetic resonance imaging for differentiation of nonocclusive intracranial vasculopathies. Stroke 48:3026-3033, 2017

16. Power S, Matouk C, Casaubon LK, Silver FL, Krings T, Mikulis DJ, et al: Vessel wall magnetic resonance imaging in acute ischemic stroke: effects of embolism and mechanical thrombectomy on the arterial wall. Stroke 45:2330-2334, 2014

17. Reilly C, Amidei C, Tolentino J, Jahromi BS, Macdonald RL: Clot volume and clearance rate as independent predictors of vasospasm after aneurysmal subarachnoid hemorrhage. J Neurosurg 101:255-261, 2004

18. Samuels OB, Joseph GJ, Lynn MJ, Smith HA, Chimowitz MI: A standardized method for measuring intracranial arterial stenosis. AJNR Am J Neuroradiol 21:643-646, 2000

19. Vergouwen MD, Vermeulen M, van Gijn J, Rinkel GJ, Wijdicks EF, Muizelaar JP, et al: Definition of delayed cerebral ischemia after aneurysmal subarachnoid hemorrhage as an outcome event in clinical trials and observational studies: proposal of a multidisciplinary research group. Stroke 41:2391-2395, 2010
20. Xu WH, Li ML, Niu JW, Feng F, Jin ZY, Gao S: Intracranial artery atherosclerosis and lumen dilation in cerebral smallvessel diseases: a high-resolution MRI study. CNS Neurosci Ther 20:364-367, 2014

\section{Disclosures}

Mr. Hippe: research grants for statistical work on other projects from GE Healthcare, Philips Healthcare, Toshiba America Medical Systems, and Siemens Medical Solutions USA.

\section{Author Contributions}

Conception and design: Mossa-Basha, Huynh. Acquisition of data: Mossa-Basha, Huynh, Morton, Levitt. Analysis and interpretation of data: Mossa-Basha, Huynh, Fata. Drafting the article: Mossa-Basha, Huynh. Critically revising the article: all authors. Approved the final version of the manuscript on behalf of all authors: Mossa-Basha. Statistical analysis: Hippe. Administrative/ technical/material support: Mossa-Basha. Study supervision: Mossa-Basha.

\section{Supplemental Information}

\section{Previous Presentations}

Portions of this work were presented at the ASNR 55th Annual Meeting, Long Beach, California, April 26, 2017.

\section{Current Affiliations}

Dr. Huynh: Department of Diagnostic Imaging, QE II Health Sciences Centre, Halifax, Nova Scotia, Canada.

\section{Correspondence}

Mahmud Mossa-Basha: University of Washington, Seattle, WA. mmossab@uw.edu. 